\title{
Effect of metformin on the anti-Müllerian hormone level in infertile women with polycystic ovarian syndrome
}

Fatemeh Foroozanfard ${ }^{1}$, Mansooreh Samimi ${ }^{2}$, Kosar Hosseini Almadani $^{3}$, Mojtaba Sehat ${ }^{3}$

${ }^{1}$ OBS\& GYN- Infertility Fellowship, Associate Professor, Gametogenesis Research Center, Kashan University of Medical Sciences, Kashan, Iran

${ }^{2}$ OBS\& GYN, Associate Professor Gametogenesis Research Center, Kashan University of Medical Sciences, Kashan, Iran

${ }^{3}$ Faculty of Medicine, Kashan University of Medical Sciences, Kashan, Iran

\section{Type of article: Original}

\begin{abstract}
Background: Polycystic ovary syndrome (PCOS) is the major cause of anovulatory infertility in women. The level of serum Anti-Mullerian Hormone (AMH) in patients can be 2-3 times higher than in healthy women. The aim of this study was to assess the effect of metformin on AMH level in PCOS patients suffering from infertility.

Methods: In this pre and post clinical trial, 30 infertile patients with PCOS were enrolled according to the Rotterdam criteria. The serum AMH level was recorded before and after 8 weeks of treatment with metformin (1500 mg daily). We used SPSS version 17 and paired samples t-test, multiple linear regression and ANCOVA test for data analysis.

Results: Serum AMH level was significantly decreased after 8 weeks of treatment with metformin $[10 \pm 3.75$ $(\mathrm{ng} / \mathrm{ml})$ versus $7.8 \pm 3.7(\mathrm{ng} / \mathrm{ml})]$ ( $\mathrm{p}=0.008,95 \%$ CI: 0.60-3.75). Also, AMH level change was directly associated to BMI in PCOS patients. In other words, in these patients, a higher BMI led to more decrease in AMH level after metformin treatment.

Conclusion: Eight weeks' treatment with metformin would significantly decrease AMH. AMH level change was directly associated to BMI.

Clinical trial registration: The trial was registered at the Iranian Registry of Clinical Trials (http://www.irct.ir) with the Irct ID: 201403132967N5

Funding: This study was funded by the Department of Gynecology and Obstetrics, School of Medicine, Kashan University of Medical Sciences (Grant Number: 9322).

Keywords: Metformin, Anti-Mullerian hormone, Polycystic ovary syndrome, Infertility
\end{abstract}

\section{Introduction}

Polycystic ovary syndrome (PCOS) is the major cause of endocrine disease and anovulatory infertility in women. PCOS is characterized by impaired ovulation, and accumulation of preantral and small antral follicles in the ovaries. It is proposed that insulin resistance and hyperinsulinemia are the main pathogenesis of anovulation and infertility in PCOS patients (1). Anti-Mullerian hormone (AMH) is a dimeric glycoprotein which is a member of transforming growth factor B (TGF-B). AMH is produced exclusively by the granulosa cells of primary, preantral and small antral follicles in women, and the serum concentration of this hormone reflects the ovarian follicular reservoir. Studies have shown that AMH level in women with PCOS are 2-3 times higher than in healthy women $(2,3)$. Metformin is the most common antihyperglycemic agent, recently introduced for treatment of women with PCOS. Some studies have attempted to investigate the AMH serum level during treatment with metformin in patients with PCOS (4). In a study by Fleming et al. (5), they found significant decrease in AMH after 8 months of the metformin administration in PCOS women. Neagu et al. (6) demonstrated that ovulation was restored in all the PCOS patients after 2 months

\section{Corresponding author:}

Kosar Hosseini Almadani, Faculty of Medicine, Kashan University of Medical Sciences, Kashan, Iran.

Tel: +98.9122532998, Fax: +98.55545057, Email: k.hosseini145663@gmail.com

Received: January 21, 2016, Accepted: August 01, 2016, Published: December 2017

iThenticate screening: June 15, 2016, English editing: November 10, 2017, Quality control: November 12, 2017

(c) 2017 The Authors. This is an open access article under the terms of the Creative Commons Attribution-NonCommercialNoDerivs License, which permits use and distribution in any medium, provided the original work is properly cited, the use is non-commercial and no modifications or adaptations are made. 
of treatment with Metformin ( $850 \mathrm{mg} /$ two times per day) and also, the serum level of AMH was significantly decreased. Paradoxically, Grigorian et al. (7) showed that serum AMH concentration did not change significantly after 6 months of metformin therapy $(850 \mathrm{mg} / \mathrm{two}$ times per day). Oral antihyperglycemic agents suppress total androstenedione and increase the follicular growth and ovulation in women with PCOS. Numerous studies have proved that metformin induces regular menstrual cycles and enhances ovulation in women with PCOS. But the impact of metformin on serum AMH level in these patients is still ambiguous (8). The serum AMH level is more reliable for evaluation of ovarian function and ovarian pool size compared to other markers such as inhibin, estradiol and follicle stimulating hormone (4). On these grounds, determination of the serum AMH changes during treatment with metformin is important for understanding the effect of this treatment on ovarian function. The aim of this study was to assess the effect of metformin treatment on serum AMH in PCOS infertile women.

\section{Material and Methods \\ 2.1 Participants}

This study was a pre and post study performed between October 2013 and November 2014. Thirty infertile PCOS women attending the infertility departments of Shahid Beheshti Hospital in Kashan, Isfahan, Iran, were invited to participate in the study. Written informed consent was acquired from all participants. Age, weight, height, menstrual pattern, presence of hirsutism, and drug history was recorded for each patient.

\subsection{Eligibility criteria}

Inclusion criteria for this study were: PCOS, age (18-35 years); no contraindications for oral administration of metformin, no administration of metformin within the previous four months, no administration of oral contraception within two months prior to the study, lack of thyroid dysfunction, hyperprolactinemia, diabetes mellitus, late-onset or congenital adrenal hyperplasia; and no medication affecting gonadal or adrenal function, carbohydrate or lipid metabolism. Exclusion criteria was metformin intolerance.

\subsection{Diagnostic and laboratory}

PCOS, was diagnosed based on the Rotterdam consensus statements criteria, and included at least two of the following three characteristics: menstrual irregularity due to oligo and/or anovulation (having an interval of $>35$ days between menstrual periods and/or amenorrhea, described as the absence of vaginal bleeding for at least six months), polycystic ovaries morphology on ultrasound exam (12 or more small follicles in an ovary), and clinical symptoms or biochemical markers of hyperandrogenism and exclusion of other etiologies (9). Clinical hyperandrogenism was evaluated by the self-reported degree of hirsutism using the modified Ferriman Gallwey (mF-G) scoring method.

\subsection{Sample size}

The sample size was determined based on findings of Neagu et al. (6) using the following formula: $n=\left(Z_{1-\alpha / 2}-Z_{1-\beta}\right)^{2}$ $\left(\mathrm{SD}_{1}+\mathrm{SD}_{2}\right)^{2} / \mathrm{d}^{2}$, where: $\alpha=0.05,1-\beta$ (Power) $=0.80, \mathrm{SD} 1=0.99$ (standard deviation of AMH level before intervention), $\mathrm{SD} 2=0.46$ (standard deviation of AMH level after intervention), $\mathrm{d}=8.99-6.28$ (difference AMH level before and after intervention). The calculated sample size was 25 .

\subsection{Research ethics}

The present study was accepted by the Ethics Committee of Kashan University of Medical Sciences (KUMS). All women signed written informed consent. This trial was registered on the Iranian website (www.irct.ir) for registration of clinical trials (IRCT code: IRCT201403132967N5)

\subsection{Study design}

First, the socio-demographic and clinical information was recorded for each patient. Height and weight were measured and body mass index (BMI) was calculated as weight $(\mathrm{kg})$ divided by height squared $\left(\mathrm{m}^{2}\right)$. All measurements were done by the same operator, to reduce subjective errors. Transvaginal ultrasound was also carried out to detect early ovarian morphology. The baseline level of FSH, LH, AMH, TSH, FBS and Prolactin was measured using $10 \mathrm{ml}$ of patient's blood plasma. For all patients, metformin was administrated $500 \mathrm{mg} /$ day in the first week, $1000 \mathrm{mg} /$ day in the second week, and $1500 \mathrm{mg} /$ day in the third week. Treatment with metformin continued for 8 weeks with the aim of ovulation occurrence. AMH level (ng/ml) was measured after 8 weeks. Obtained results were compared with those of the first tests. Patients were divided in to their groups according to body mass indexes. Patients with BMI $\leq 25$ were considered normal, BMI 25-29.9 overweight and BMI $\geq 30$ were obese patients. 


\subsection{Biochemical analysis}

Blood plasma $(10 \mathrm{ml})$ was taken to measure the baseline level of FSH, LH, AMH, TSH, FBS and Prolactin. AMH, FSH, LH, and Prolactin were quantified using an ELISA kit. Fasting plasma glucose was measured by the glucose oxidase method, and TSH was measured by immunofluorescence assay.

\subsection{Statistical analysis}

Data was presented as numbers and percentage, unless otherwise indicated. AMH level comparison before and after intervention was carried out using paired-samples t-test. Multiple Linear Regression test was applied to identify variables which contributed to the AMH level. P-value less than 0.05 was considered statistically significant. Statistical analysis was carried out using SPSS version 13.0 (SPSS; SPSS Inc., Chicago, IL, USA).

\section{Results}

Forty five infertile PCOS women were enrolled in the study. Two patients were excluded due to drug intolerance, four patients due to pregnancy during treatment with metformin, and nine patients decided to exit the study, finally, thirty patients continued the study. The mean $( \pm \mathrm{SD})$ age and BMI of patients were $25.2 \pm 4.2$ years and $26.2 \pm 3.8$ $\mathrm{kg} / \mathrm{m}^{2}$, respectively (Table 1). Level of AMH 2 months after metformin treatment significantly decreased in the overweight group. Obese subjects had more reduction in AMH (Table 2). The mean $( \pm \mathrm{SD})$ serum level of AMH before intervention was $10 \pm 3.7 \mathrm{ng} / \mathrm{ml}$, and was significantly decreased after 8 weeks of metformin treatment $(7.8 \pm 3.7 \mathrm{ng} / \mathrm{ml})(\mathrm{diff}=2.18 ; 95 \% \mathrm{CI}: 0.60-3.75) \mathrm{p}=0.008$. In the PCOS population, there was an inverse correlation between BMI and AMH, which was not significant $(\beta=-0.34, \mathrm{p}=0.85)$. According to regression analysis, AMH level change was directly associated to BMI $(\beta=-0.345,95 \%$ CI: $-0.75-0.03, p=0.06)$. In other words a higher BMI led to more decrease in $\mathrm{AMH}$ level after metformin treatment.

Table1. Clinical and hormonal data in women with PCOS and infertility

\begin{tabular}{|l|l|l|l|}
\hline Variable & Mean & CI 95\% & Normal range of laboratory \\
\hline Age $($ year $)$ & 25.3 & $23.8-26.8$ & - \\
\hline BMI $(\mathrm{kg} / \mathrm{m} 2)$ & 26.1 & $24.7-27.5$ & - \\
\hline LH $(\mathrm{mIU} / \mathrm{ml})$ & 8.1 & $6.6-9.6$ & $2.4-12.6$ \\
\hline FSH $(\mathrm{mIU} / \mathrm{ml})$ & 4.8 & $3.7-5.9$ & $3.5-12.5$ \\
\hline FBS $(\mathrm{mg} / \mathrm{dl})$ & 91.4 & $87.6-95.3$ & $70-110$ \\
\hline AMH $(\mathrm{ng} / \mathrm{ml})$ & 10 & $8.6-11.4$ & $2.2-6$ \\
\hline Prolactin $(\mathrm{ng} / \mathrm{ml})$ & 19.1 & $16.3-22.06$ & $2.8-29.2$ \\
\hline TSH $(\mu \mathrm{IU} / \mathrm{ml})$ & 3.06 & $2.3-3.7$ & $0.3-5.5$ \\
\hline
\end{tabular}

Table 2. Change of AMH level after 2 months' treatment with metformin by BMI

\begin{tabular}{|c|c|c|c|c|c|}
\hline \multirow[t]{2}{*}{ BMI } & \multicolumn{2}{|c|}{ AMH (before) } & \multicolumn{2}{|c|}{ AMH (2 month later) } & \multirow[t]{2}{*}{ p-value } \\
\hline & Mean & SD & Mean & SD & \\
\hline Normal & 9.91 & 2.80 & 10.27 & 4.18 & 0.721 \\
\hline Overweight & 9.49 & 4.13 & 6.31 & 2.01 & 0.01 \\
\hline Obesity & 12.25 & 4.44 & 7.75 & 5.85 & 0.068 \\
\hline
\end{tabular}

\section{Discussion}

The effect of metformin on serum AMH levels in patients with polycystic ovary syndrome (PCOS) is ambiguous. In this study, we examined the effect of metformin on AMH serum levels in PCOS patients during, before- and after study. The baseline assessment of AMH serum level in PCOS women before treatment with metformin, confirms the previous findings that AMH serum levels are 2-3 times higher in PCOS women than in healthy women $(2,10)$. This hormone is mainly secreted by granulosa cells of pre-antral and small antral follicles. Therefore, the greater quantities of this hormone in circulation may be due to a combination of increased specific secretion and increased follicle counts $(2,3)$. Animal studies have shown that insulin promotes primordial the activation of primordial follicles (11). However, the studies on hypophysectomized animals suggested that pituitary gonadotropins are not totally necessary for the activation of primordial follicles (12). Furthermore, hyperinsulinemia is a common factor which may be associated with PCOS that play a role in anovulation, hyperandrogenism, and AMH increase in PCOS (13). AMH negatively regulates primordial follicle activation (14). Therefore, insulin-sensitizing drugs, including metformin are used for most women with PCOS (5). In our study, we administrated metformin in PCOS patients and our findings were consistent with Fleming et al. (5). They found significant decrease in AMH after eight months of 
metformin treatment. Also, Piltonen et al. (4) have shown that AMH serum level significantly decreased after metformin treatment (1500mg /day) for six months in PCOS women. Paradoxically, Grigorian et al. (7) have shown that AMH concentration during metformin therapy did not change significantly. The study included 40 obese women with PCOS. All patients received metformin hydrochloride administrated, one tablet (850mg) two times a day for six months. The concentration of AMH did not change significantly during the treatment. Also, Nascimento et al. found that metformin treatment leads to a significant decrease in serum insulin and testosterone levels. However, no alternation in AMH level was observed after treatment (15). Recently, Madsen et al. (2015) suggested that the decrease in AMH level after 6 months of metformin treatment is, overall, not significant (8). In our study, a dose of $1500 \mathrm{mg}$ metformin was administered daily for two months, while the prescribed dose was higher and the treatment duration was longer in previous studies. Our study has suggested that the dose of $1500 \mathrm{mg}$ metformin daily for two months could be effective enough. Therefore, it could be theorized that metformin may have an effect on AMH levels by means of influencing insulin levels in PCOS patients. According to our study in PCOS population type 1, there was not significant correlation between BMI and AMH. A study in 2015 by Maya Krisman et al. (16) showed that although there was no correlation between BMI and AMH level in the general population, in the PCOS population, AMH was reversely correlated with BMI. This difference may be due to the small sample size in our study. Some limitation must be considered in the interpretation of our findings: 1) the small sample size led to an inadequately powered analysis for the major confounder, 2) lack of control group, 3) we did not examine the effect of metformin on serum androgen due to limited funding. It is recommended that further studies be conducted with more population and multicenter design. In conclusion, metformin is able to decrease AMH in PCOS, presumably by an insulin-dependent mechanism of action.

\section{Conclusions}

Our findings showed that serum AMH level was significantly decreased after 8 weeks' treatment with metformin. AMH level change was directly associated to BMI. Metformin may have an effect on AMH level by means of influencing the insulin level in PCOS patients. It is recommended that further studies be conducted with more population and multicenter design, and with a control group.

\section{Acknowledgments:}

This article was extracted from the thesis by Kosar Hosseini Almadani. The authors express thanks for the financial support of the Vice Chancellor of Research and Technology, Kashan University of Medical Sciences. The authors also thank Dr. Mirzaee for his cooperation.

\section{Clinical trial registration:}

The trial was registered at the Iranian Registry of Clinical Trials (http:/www.irct.ir) with the Irct ID: 201403132967 N5.

\section{Funding:}

This study was funded by the Department of Gynecology and Obstetrics, School of Medicine, Kashan University of Medical Sciences (Grant Number: 9322).

\section{Conflict of Interest:}

There is no conflict of interest to be declared.

\section{Authors' contributions:}

All authors contributed to this project and article equally. All authors read and approved the final manuscript.

\section{References:}

1) Nardo LG, Yates AP, Roberts SA, Pemberton P, Laing I. The relationships between AMH, androgens, insulin resistance and basal ovarian follicular status in non-obese subfertile women with and without polycystic ovary syndrome. HumReprod. 2009; 24(11): 2917-23. doi: 10.1093/humrep/dep225. PMID: 19617605.

2) Pigny P, Merlen E, Robert Y, Cortet-Rudelli C, Decanter C, Jonard S, et al. Elevated serum level of antimullerian hormone in patients with polycystic ovary syndrome: relationship to the ovarian follicle excess and to the follicular arrest. J Clin Endocrinol Metab. 2003; 88(12): 5957-62. doi: 10.1210/jc.2003-030727. PMID: 14671196. 
3) Laven JS, Mulders AG, Visser JA, Themmen AP, De Jong FH, Fauser BC. Anti-Mullerian hormone serum concentrations in normoovulatory and anovulatory women of reproductive age. J Clin Endocrinol Metab. 2004, 89(1): 318-23. doi: 10.1210/jc.2003-030932. PMID: 14715867.

4) Piltonen T, Morin-Papunen L, Koivunen R, Perheentupa A, Ruokonen A, Tapanainen JS. Serum antiMullerian hormone levels remain high until late reproductive age and decrease during metformin therapy in women with polycystic ovary syndrome. Hum Reprod. 2005; 20(7): 1820-6. doi: 10.1093/humrep/deh850. PMID: 15802325.

5) Fleming R, Harborne L, MacLaughlin DT, Ling D, Norman J, Sattar N, et al. Metformin reduces serum mullerian-inhibiting substance levels in women with polycystic ovary syndrome after protracted treatment. Fertil Steril. 2005; 83(1): 130-6. doi: 10.1016/j.fertnstert.2004.05.098. PMID: 15652898.

6) Neagu M, Cristescu C. Anti-Mullerian hormone--a prognostic marker for metformin therapy efficiency in the treatment of women with infertility and polycystic ovary syndrome. J Med Life. 2012; 5(4): 462-4. PMID: 23346251, PMCID: PMC3539844.

7) Grigoryan O, Absatarova J, Andreeva E, Melnichenko G, Dedov I. Effect of metformin on the level of antiMullerian hormone in therapy of polycystic ovary syndrome in obese women. Minerva Ginecol. 2014; 66(1): 85-9. PMID: 24569407.

8) Madsen HN, Lauszus FF, Trolle B, Ingerslev HJ, Torring N. Impact of metformin on anti-Mullerian hormone in women with polycystic ovary syndrome: a secondary analysis of a randomized controlled trial. Acta Obstet Gynecol Scand. 2015; 94(5): 547-51. doi: 10.1111/aogs.12605. PMID: 25736975.

9) Adams J, Polson DW, Franks S. Prevalence of polycystic ovaries in women with anovulation and idiopathic hirsutism. Br Med J (Clin Res Ed). 1986; 293(6543): 355-9. PMID: 3089520, PMCID: PMC1341046.

10) Fallat ME, Siow Y, Marra M, Cook C, Carrillo A. Mullerian-inhibiting substance in follicular fluid and serum: a comparison of patients with tubal factor infertility, polycystic ovary syndrome, and endometriosis. Fertil Steril. 1997; 67(5): 962-5. PMID: 9130910.

11) Kezele PR, Nilsson EE, Skinner MK. Insulin but not insulin-like growth factor-1 promotes the primordial to primary follicle transition. Mol Cell Endocrinol. 2002; 192(1-2): 37-43. PMID: 12088865.

12) Gulyas BJ, Hodgen GD, Tullner WW, Ross GT. Effects of fetal or maternal hypophysectomy on endocrine organs and body weight in infant rhesus monkeys (Macacamulatta): with particular emphasis on oogenesis. Biol Reprod. 1977; 16(2): 216-27. PMID: 401655.

13) Dunaif A. Insulin resistance and the polycystic ovary syndrome: mechanism and implications for pathogenesis. Endocr Rev. 1997; 18(6): 774-800.

14) Knight PG, Glister C. TGF-beta superfamily members and ovarian follicle development. Reproduction. 2006; 132(2): 191-206.

15) Nascimento AD, Silva Lara LA, Japur de Sá Rosa-e-Silva AC, Ferriani RA, Reis RM. Effects of metformin on serum insulin and anti-Mullerian hormone levels and on hyperandrogenism in patients with polycystic ovary syndrome. Gynecol Endocrinol. 2013; 29(3): 246-9. doi: 10.3109/09513590.2012.736563. PMID: 23194004.

16) Kriseman M, Mills C, Kovanci E, Sangi-Haghpeykar H, Gibbons W. Antimullerian hormone levels are inversely associated with bodymass index (BMI) in women with polycystic ovary syndrome. J Assist Reprod Genet. 2015; 32(9): 1313-6. doi: 10.1007/s10815-015-0540-0. PMID: 26238387, PMCID: PMC4595400. 